\title{
A Case of Bardet Biedl Syndrome Presenting With Insulin Dependent Diabetes Mellitus
}

\author{
Authors \\ Shashwat Bhattacharyya ${ }^{1}$, Aditi Maitra ${ }^{2}$, Anuria De $^{1}$, Ankit Shalikram Gedam ${ }^{1}$ \\ ${ }^{1}$ Department of Ophthalmology, Calcutta National Medical College, Kolkata, India \\ ${ }^{2}$ Dept of Clinical \& Experimental Pharmacology, Calcutta School of Tropical Medicine, Kolkata, India
}

\begin{abstract}
Purpose: To report an extremely rare association of Bardet Biedl syndrome (BBS) with insulin dependent diabetes mellitus.

Case Report: A 14 year old male child presented to the ophthalmology clinic with complaint of poor vision of both eyes since early childhood. The parents also gave a history of mental retardation, extra digits in both hands and feet, poorly developed genitals and obesity. The child was previously diagnosed to be suffering from insulin dependent (IDDM). Ocular examination revealed poor vision in both eyes, pigmentary retinopathy with consecutive optic atrophy and macular dystrophic changes. This was established as rod cone dystrophy on $\mathrm{mf-ERG}$. Systemic examination revealed that the child had obesity, post-axial polydactyly, short stature, micropenis, small testes bilaterally and psychometric testing showed intellectual impairment.
\end{abstract}

Conclusion: This report of rod-cone dystrophy associated with IDDM significantly expands the spectrum of $B B S$ phenotype.

Keyword: Bardet Biedl syndrome; Insulin dependent diabetes mellitus; retinitis pigmentosa; rod cone dystrophy.

\section{INTRODUCTION}

Bardet-Biedl syndrome (BBS) is a rare multisystem disorder, arising out of basal body dysfunction secondary to ciliopathy. ${ }^{1}$ The prevalence ranges from 1:160000 in Northern America and Europe to 1:13500 in isolated communities in Kuwait and Newfoundland, owing to a high degree of consanguinity in these populations. ${ }^{2}$ The main clinical features are rod-cone dystrophy with retinal degeneration, postaxial polydactyly, truncal obesity, male hypogonadism, renal anomalies and mental retardation. $^{2}$ There may be secondary features such as cardiac dysfunction, diabetes mellitus, gastrointestinal function anomalies or speech anomalies. The development of type 2 diabetes is prevalent among patients and it may be related to the level of obesity. ${ }^{3}$ The association of BBS with type 1 or IDM mellitus (IDDM) is an extremely rare occurrence and as such has been seldom reported in literature. ${ }^{4}$ We therefore present this rare association as a case report.

\section{CASE REPORT}

A fourteen year old male child presented to the ophthalmology outpatient department of a tertiary care hospital with complaints of very poor vision of 
both eyes since early childhood. The child was born out of a second degree consanguineous marriage. The parents also gave a history of mental retardation, extra digits in both hands and feet (figure 1), poorly developed genitals (figure 2) and obesity. The presence of IDDM was narrated by the parents. It was diagnosed at the age of seven years following an episode of hospitalization for extreme fatigue. The child was on insulin therapy since then. He had an elder sister who was without any significant medical condition. The parents too did not give any history of significant medical conditions.

A thorough ocular examination was carried out which revealed a distant visual activity of 3/60 in the right eye (RE) and 1/60 in the left eye (LE). Both the eyes had a refractive error of -2.75 Diopters spherical error. Extraocular movements were full in all directions of gaze. Intra ocular pressure was $14 \mathrm{~mm}$ of $\mathrm{Hg}$ (Central Corneal Thickness: $538 \mu \mathrm{m}$ ) in $\mathrm{RE}$ and $16 \mathrm{~mm}$ of $\mathrm{Hg}$ (Central Corneal Thickness: $542 \mu \mathrm{m}$ ) in LE. The anterior segments were normal in both eyes on slit lamp examination. Gonioscopy showed open angles in all quadrants in both eyes.

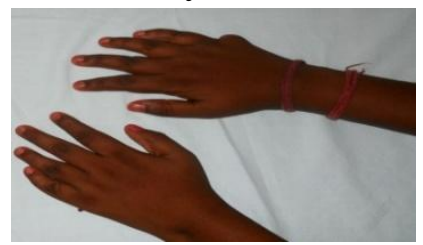

Figure 1: Post axial polydactyly

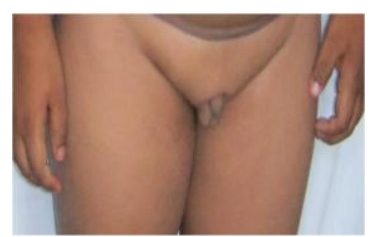

Figure 2: Poorly developed genitals

Fundus examination (figures $3 \mathrm{a} \& 3 \mathrm{~b}$ ) revealed optic atrophy, attenuated retinal arterioles, cellophaning of macula and a myopic tesselated background with plenty of pigmented bony spicules in the mid periphery (figures $4 a \& 4 b$ ). There were dystrophic macular changes in both the eyes. OCT macula of both eyes revealed reduced foveal thickness with disruption of normal contour (figures 5a \& 5b). A full field multifocal ERG revealed that both rod (scotopic) and cone (photopic) responses were extinguished.
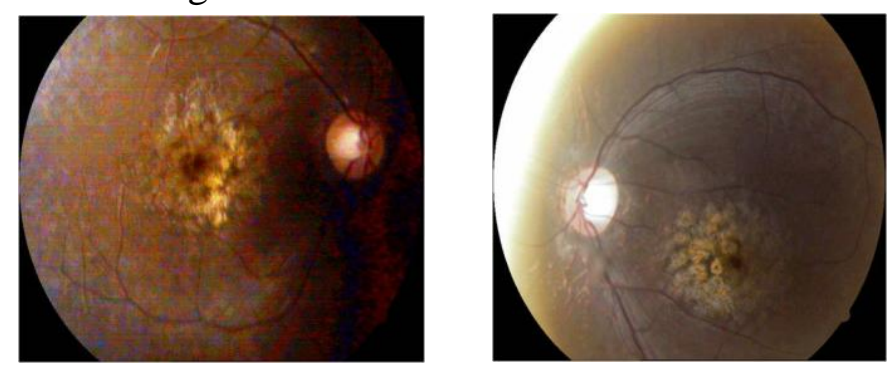

Figure 3a \& 3b: Fundus photos of RE and LE showing opti,c atrophy and arteiolar attenuation and epiretinal membrane
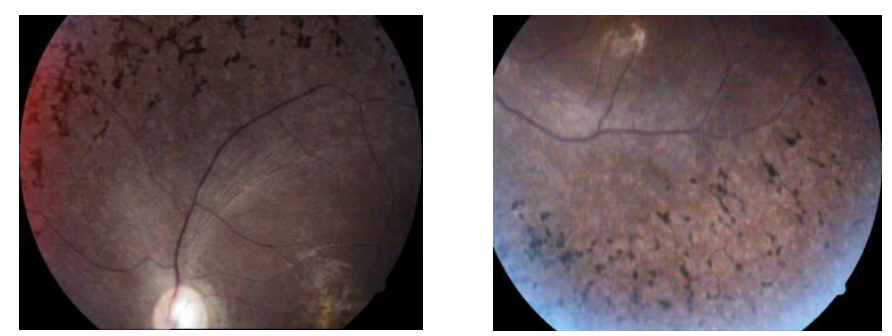

Figure 4a \& 4b: Peripheral fundus showing bony spicular pigmentation

On systemic examination it was seen that the child had post-axial polydactyly, body weight of 67 kilograms, short stature $(126 \mathrm{~cm})$, micropenis $(2.5$ $\mathrm{cm})$, Tanner stage I testes bilaterally and truncal obesity. Psychometric testing revealed intellectual impairment. The child was diagnosed to be suffering from hypogonadotrophic hypogonadism as the levels of FSH, LH and testosterone were pre pubertal. Multisystem examination including renal (USG-KUB), cardiovascular (2D Echocardiography) and E.N.T. (BAER \& Olfactory tests) revealed no significant anomalies.
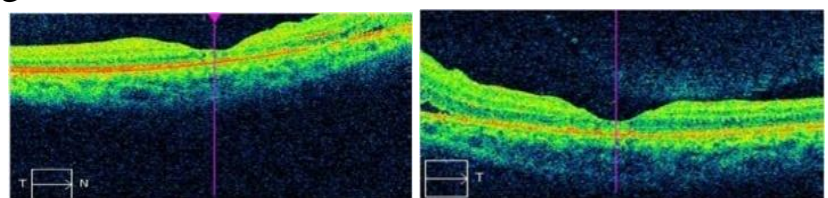

Figure 5a \& 5b: OCT macula of RE \& LE

\section{DISCUSSION}

The manifestations of BBS were initially described by Bardet in 1920 and Biedl in 1922., It is differentiated from a condition reported by Lawrence and Moon in 1865, which is characterised by retinal pigmentary degeneration, hypogonadism 
and mental retardation which occur in conjunction with progressive spastic paraparesis and distal muscle weakness, but without polydactyly. ${ }^{7,8}$ BBS is genetically heterogeneous, with 21 BBS genes identified to date. ${ }^{9}$ Although the cellular mechanisms that underlie BBS remain unclear, it is now clear that all of the known BBS proteins are components of the centrosome and the basal body and have an impact on ciliary transport. ${ }^{10}$

The presence of retinitis pigmentosa along with diabetes mellitus is reported in BBS, Kearns-Sayre syndrome, Alström syndrome and Wolfram syndrome. Kearns-Sayre syndrome is a mitochondrial disease differentiated from BBS by the presence of cardiac conduction defects, hyperaldostronism and external ophthalmoplegia. ${ }^{11}$ In Alström syndrome cognitive function is maintained, post axial polydactyly is not seen; dilated cardiomyopathy and hepatic dysfunction are diagnostic criterion which separates it from BBS. ${ }^{12}$ Wolfram syndrome is a rare autosomal recessive neurodegenerative disease which is characterised by diabetes insipidus, diabetes mellitus, optic atrophy and deafness with few reported associations of retinitis pigmentosa. ${ }^{13}$ BBS exhibits oligogenic inheritance, there have been reports of some patients having 3 mutations in 2 unique BBS loci, which may further interact and thus change the phenotype. ${ }^{14}$ Given this pleomorphic genotypical nature of BBS and the strong effects of genetic mutations on the causality of IDDM, the association between the two is being reported as an expansion of the spectrum of BBS phenotype. This report of IDDM is another unique and extremely rare occurrence. To date, only Escallon et al have reported a family with the BBS and DM. ${ }^{4}$ Three siblings were examined. The 18 year-old brother had obesity in that report with mental retardation, retinitis pigmentosa and IDDM. Further genetic studies are required to substantiate and establish the causal association of BBS and IDDM.

\section{CONCLUSION}

BBS is associated with marked phenotypic and genotypic variability, which complicates diagnosis and genetic counseling. The present patient with rod-cone dystrophy associated with IDDM is a significant addition to the phenotype spectrum. Recognition of these unusual phenotypes and an understanding of the underlying pathophysiology are crucial to the diagnosis and management of these patients and the formulation of any future therapy for BBS.

\section{REFERENCE}

1. Runge P, Calver D, Marshall J, Taylor D. Histopathology of mitochondrial cytopathy and the Laurence-Moon-Biedl syndrome. British journal of ophthalmology. 1986 Oct 1;70(10):782-96.

2. Beales PL, Elcioglu N, Woolf AS, Parker D, Flinter FA. New criteria for improved diagnosis of Bardet-Biedl syndrome: results of a population survey. Journal of medical genetics. 1999 Jun 1;36(6):437-46.

3. Forsythe E, Beales PL. Bardet-Biedl syndrome. European journal of human genetics. 2013 Jan 1;21(1):8-13.

4. Escallon F, Traboulsi EI, Infante R. A family with the Bardet-Biedl syndrome and diabetes mellitus. Archives of Ophthalmology. 1989 Jun 1;107(6):855-7.

5. Bardet G. Sur un syndrome d'obèsite infantile avec polydactyly et rétinite pigmentaire (Doctoral dissertation, Thesis, University of Paris, France).

6. Biedl A. Ein Geschwisterpaar mit adiposogenitaler Dystrophie. Dtsch Med Wochenschr. 1922;48:1630.

7. Laurence JZ, Moon RC. Four cases of "retinitis pigmentosa" occurring in the same family, and accompanied by general imperfections of development. Ophthalmol Rev1866;2:32-41.

8. Harnett JD, Green JS, Cramer BC, et al. The spectrum of renal disease in LaurenceMoon-Biedl syndrome. N Engl J Med 1988;319:615-18.

9. Heon E, Kim G, Qin S, Garrison JE, Tavares E,Vincent A et al. Mutations in C8ORF37 
cause Bardet Biedl syndrome (BBS21).

Hum Mol Genet. 2016 Mar 22. pii: ddw096

10. Hichri H, Stoetzel C, Laurier V, Caron S, Sigaudy S, Sarda P, Hamel C, MartinCoignard D, Gilles M, Leheup B, Holder M. Testing for triallelism: analysis of six BBS genes in a Bardet-Biedl syndrome family cohort. European journal of human genetics. 2005 May 1;13(5):607-16.

11. The clinical diagnosis and molecular genetics of kearns-sayre syndrome: a complex mitochondrial encephalomyopathy. Maceluch JA, Niedziela M Pediatr Endocrinol Rev. 2006 Dec-2007 Jan; 4(2):117-37

12. Alstrom syndrome (OMIM 203800): a case report and literature review. Joy T, Cao H, Black G, Malik R, Charlton-Menys V, Hegele RA, Durrington PN Orphanet J Rare Dis. 2007 Dec 21; 2():49.

13. Ophthalmologic findings in fifteen patients with Wolfram syndrome. Al-Till M, Jarrah NS, Ajlouni KM Eur J Ophthalmol. 2002 Mar-Apr; 12(2):84-8.

14. Katsanis N, Ansley SJ, Badano JL, Eichers ER, Lewis RA, Hoskins BE, Scambler PJ, Davidson WS, Beales PL, Lupski JR. Triallelic inheritance in Bardet-Biedl syndrome, a Mendelian recessive disorder. Science. 2001 Sep 21;293(5538):2256-9. 